with resistance to zoliflodacin, all located in the gyrB gene: $\mathrm{D} 429 \mathrm{~N}, \mathrm{~K} 450 \mathrm{~N}$, and K450T. To determine the prevalence of those mutations within $\mathrm{N}$. gonorrhoeae whole genome sequences, we searched PathogenWatch, an online global database for genomic surveillance.

Methods We downloaded all available N. gonorrhoeae genomes from PathogenWatch (https://pathogen.watch/) on November 17th, 2020. The gyrB and gyrA gene sequences were obtained from the EzBioCloud database. We used the $\mathrm{N}$. gonorrhoeae FA 1090 genome as our reference, and the wildtype gyrA sequence was included in our search as a control. BLAST $(2.2 .26+)$ was used to query each of the two genes to the reference genomes with a $60 \%$ identity/length threshold value. Biopython BLAST IO package was used to parse the result, and subsequent DNA translation to protein was conducted. The counts of the mutations of interest were measured using in-house python code, which generates the counts of different amino acids with given position value. Some fragmented genes were manually validated after the protein alignment using MUSCLE (3.8.31).

Results In total, $12,943 \mathrm{~N}$. gonorrhoeae genomes were searched. No sequences contained the D429N, K450N, or $\mathrm{K} 450 \mathrm{~T}$ mutations in gyrB. One sequence was identified with a D429V mutation, a mutation previously unreported but similar to D429N. In total, 5395 sequences harbored the gyrA S91F mutation, while 5392 (99.9\%) of those sequences were correctly identified by PathogenWatch. The three gyrA sequences with discrepancies were confirmed manually.

Conclusion Of the 12,943 publicly available N. gonorrhoeae genomes on the PathogenWatch database, none were found to harbor mutations in gyrB known to be associated with zoliflodacin resistance. When zoliflodacin becomes clinically available, resistance due to known mutations in gyrB is likely to be rare.

\section{P037 HIV TRANSMISSION AND PREVIOUS PREP AWARENESS AND USE IN MEN WHO HAVE SEX WITH MEN (MSM)}

${ }^{1} \mathrm{C}$ Norcross*, ${ }^{1,2} \mathrm{D}$ Richardson. 'Brighton and Sussex University Hospitals NHS Trust, Brighton, UK; ${ }^{2}$ Brighton and Sussex Medical School, Brighton, UK

\subsection{6/sextrans-2021-sti.184}

Background HIV Pre-Exposure Prophylaxis (PrEP) has been available to men who have sex with men (MSM) via clinical trials or self-funding (online) in the UK since 2017. Despite its effectiveness and availability, we are still observing HIV transmission in MSM. This study aimed to assess prior awareness, prior usage and reasons for non-uptake of PrEP in MSM newly diagnosed with HIV.

Methods All MSM who were diagnosed with HIV at this sexual health clinic between January 2017 and September 2020 were identified and their clinical records examined for information on previous clinic attendances; PrEP discussion with a clinician and PrEP use prior to HIV diagnosis.

Results 59 MSM were diagnosed with HIV during the study period, with a median age of $42[\mathrm{IQR}=31.0-50.4] .24(41 \%)$ had attended the sexual health clinic between the time PrEP became available and HIV diagnosis. 24 (41\%) had a concurrent bacterial STI at HIV diagnosis and 23 (39\%) reported recreational drug use prior to HIV diagnosis, with $4(7 \%)$ reporting intravenous drug use. $16(27 \%)$ had prior awareness of PrEP. Of these, 1 MSM had used PrEP inconsistently due to concern about stigma and low self-perceived HIV risk, 5 were unable to access PrEP due to insufficient clinical trial spaces or barriers to self-funding, 2 cited no specific reason for non-uptake and 8 were found to be HIV positive at date of first PrEP discussion.

Conclusion The majority of MSM diagnosed with HIV had poor prior PrEP awareness. Access to PrEP was poor despite evidence of risk taking behaviour such as bacterial STIs and drug use. Barriers to uptake included lack of places in clinical trials, difficulty self-funding, low perceived risk and stigma. PrEP is now freely accessible in the UK, but continued efforts are needed to increase awareness and accessibility to further reduce HIV transmission.

\section{P038 PREVALENCE OF SYPHILIS IN PEOPLE LIVING WITH HIV/ AIDS IN THE AMERICAS: A SYSTEMATIC REVIEW AND META-ANALYSIS OF PREVALENCE STUDIES}

1,2,3 J Garcia Luna* 4 L Aguirre Martinez, ${ }^{2} \mathrm{~A}$ Smith, ${ }^{2} \mathrm{D}$ Bennett, ${ }^{1} \mathrm{M}$ Amórtegui Quinter, ${ }^{1} D$ Rebellon Sanchez, ${ }^{1} S$ Silva Peña, ${ }^{5} D$ Giron Ceron, ${ }^{1} A$ Del Castillo Pinto, ${ }^{4} D$ Vinueza, ${ }^{6} \mathrm{~J}$ Llanos Torres, ${ }^{4} \mathrm{~J}$ Zuñiga Gaitan, ${ }^{4} \mathrm{P}$ Certuche Trochez, ${ }^{4}$ I Martínez Hurtado, ${ }^{7}$ A Guerra Saavedra, ${ }^{4} \mathrm{~N}$ Sanchez Muriel. 'Centro Internacional de Entrenamiento e Investigaciones Médicas, Cali, Colombia; ${ }^{2}$ Nuffield Department of Population Health, University of Oxford, Oxford, UK; ${ }^{3}$ Universidad Icesi, Cali, Colombia; ${ }^{4}$ School of medicine, Universidad del Valle, Cali, Colombia; ${ }^{5}$ Department of biological sciences, Simon Fraser University, Canada; ${ }^{6}$ School of medicine, Universidad Icesi, Cali, Colombia; ${ }^{7}$ Universidad Libre, Cali, Colombia

\subsection{6/sextrans-2021-sti. 185}

Background Syphilis remains a public health threat, particularly in People Living with HIV/AIDS (PLWHA), due to its potential complications. The prevalence of syphilis in PLWHA in the Americas is not well characterised. Therefore, the aim of this systematic review and meta-analysis was to estimate the prevalence of syphilis in this population and to investigate sources of variation in these prevalences (PROSPERO CRD42020189246).

Methods PubMed, Embase, LILACS and Web of Science were searched for studies reporting the prevalence point of Likely Exposure to Treponema Pallidum (LETP) by treponemal tests or Likely Current Syphilis Infection (LCSI) by the combination of treponemal and non-treponemal tests, in a broadly representative sample of PLWHA in the Americas. Published studies with less than 200 PLWHA, languages other than Spanish or English, and conference abstracts were excluded. A standardised data extraction form was used. A random-effects metaanalysis was performed to obtain the pooled prevalence of LETP and LCSI with their corresponding 95\% prediction intervals $(95 \% \mathrm{PI})$. Heterogeneity was investigated by a priori defined subpopulations diagnostic algorithm and geographical region. Heterogeneity was assessed via the Cochran's Q test and I2 statistic, while Egger's test was used to assess for publication bias.

Results 25,848 records were identified, of which 49 unique studies with 67 prevalence points were included. The pooled prevalence in the Americas was $16.4 \%$ (95\%PI 2.3-49.3) for LEPT, and 7.2\% (95\%PI 0.01-25.15) for LCSI, with high heterogeneity (I2 $>75 \%$, p-value $<0.001)$. The prevalence of LETP was higher in men who have sex with men (MSM), while the prevalence of LCSI was higher in Latin America. There was no evidence of publication bias (Egger pvalue $>0.5$ ). 
Conclusions The prevalences of LETP and LCSI are high in the Americas. Public health strategies should aim to create surveillance systems of syphilis in PLWHA and assure annual screening and timely treatment.

\section{P040 IMPLEMENTING A RAPID SEXUAL HEALTH TESTING, DIAGNOSIS AND TREATMENT SERVICE: QUALITATIVE EVALUATION}

1J Horwood*, ${ }^{1} \mathrm{~A}$ Lorenc, ${ }^{1} \mathrm{E}$ Brangan, ${ }^{1} \mathrm{~J}$ Kesten, ${ }^{2} \mathrm{M}$ Croft, ${ }^{2} \mathrm{M}$ Clarke, ${ }^{1,2} \mathrm{P}$ Horner. ${ }^{1}$ University of Bristol, Bristol, UK; ${ }^{2}$ Unity Sexual Health, Bristol, UK

\subsection{6/sextrans-2021-sti.186}

Background Unity Sexual Health in Bristol UK, re-designed its service to improve access and delivery of care. This includes a Panther (Hologic Inc) system at the point of care to provide rapid STI tests, allowing Nucleic acid amplification testing results for STIs including gonorrhoea and chlamydia to be available within four hours. Previously patients waited over a week for results.

Methods A qualitative evaluation running alongside the implementation of the new service, to understand experiences, and inform its iterative development. A total of 21 members of staff and 26 patients were interviewed, and 40 hours of observations conducted of the service in operation, were analysed thematically.

Results The new service implementation required co-ordinated changes in practice across multiple staff teams. Patients also needed to make changes to how they accessed the service. Multiple small 'pilots' of process changes were necessary to find workable options. This responsive model created challenges for delivering comprehensive training/communication in advance to all staff. However, staff worked together to adjust and improve the new service, and morale was buoyed through observing positive impacts on patient care. Patients valued faster results and avoiding unnecessary treatment. They were willing to drop off samples and return for a follow-up appointment the same/next day, enabling infection-specific treatment in accordance with test results thus improving antimicrobial stewardship.

Conclusions Implementation of service changes to improve access and delivery of care in the context of stretched resources can pose challenges for staff at all levels. Early evaluation of pilots of process change, provide opportunities for prompt feedback enabling adjustment, is valued. Visibility to staff of positive impacts on patient care is important in maintaining morale. The service was acceptable to patients.

\section{P041 BARRIERS AND FACILITATORS TO ANTIRETROVIRAL THERAPY INITIATION AND ADHERENCE IN INDONESIA: HEALTH CARE PROVIDER'S PERSPECTIVES}

B Hutahaean*, S Stutterheim, K Jonas. Faculty of Psychology and Neuroscience Maastricht University, Maastricht, The Netherlands

\subsection{6/sextrans-2021-sti.187}

Background Indonesia has the fourth-largest number of new HIV diagnoses per year worldwide, is the only country in the Asia-Pacific region where HIV prevalence is increasing, and the WHO aims of $90-90-90$ has not yet been reached. It is therefore important to investigate barriers and facilitators to antiretroviral (ARV) therapy initiation and adherence. This study set out to delineate these barriers and facilitators from the perspective of health care providers.

Methods Between March and May 2020, 20 semi-structured interviews were conducted with health care providers in Indonesia. Thematic analyses were subsequently conducted to ascertain categories of barriers and facilitators to initiation and adherence.

Results Main facilitators to ARV initiation and adherence were social support; good client-provider communication; less bureaucracy or easy access to ARV; and sufficient HIV and ARV knowledge among people with HIV. Additionally, the use of euphemistic terminology for ARV was a facilitator for adherence, but not for initiation; whereas having sufficient self-care motivation, a desire to live or having health goals, and HIV status acceptance were facilitators to initiation but not adherence.

Barriers to initiation and adherence included stigma; complicated bureaucracy; insufficient health care facilities, health care coverage or ARV supply; and distance to clinics. Side effects and experiencing regimens as tedious were additional barriers to adherence; and being in denial, being asymptomatic, fatalism, and the influence of anti-ARV social media were additional barriers to initiation.

Conclusion Barriers and facilitators to initiation and adherence occur on various socio-ecological levels and should therefore be targeted on structural, interpersonal, and individual levels. Health care providers can play a key role in promoting facilitators and reducing barriers, but must be supported by national and organizational level efforts that increase access to HIV clinics and health care coverage, and decrease bureaucracy and community-level initiatives that correct myths and misinformation.

\section{P042 PREVALENCE AND ASSOCIATED FACTORS OF BEING DIAGNOSED WITH SYPHILIS AMONGST MSM ATTENDING AS SEXUAL CONTACTS OF SYPHILIS}

${ }^{1,2} \mathrm{D}$ Richardson*, ${ }^{1} \mathrm{~A}$ Pickering, ${ }^{1} \mathrm{~K}$ Nichols, ${ }^{1} \mathrm{Z}$ Buss, ${ }^{1} \mathrm{D}$ Trotman, ${ }^{1} \mathrm{C}$ Fitzpatrick, ${ }^{1} \mathrm{D}$ Williams. ${ }^{1}$ Brighton and Sussex University Hospitals NHS Trust, Brighton, UK; ${ }^{2}$ Brighton and Sussex Medical School, Brighton, UK

\subsection{6/sextrans-2021-sti.188}

Partner notification strategies have increased the number of MSM attending sexually transmitted infection (STI) clinics as sexual contacts of syphilis. Current guidelines suggest testing and consideration of presumptive antimicrobial treatment. Syphilis treatment with benzathine penicillin; requires clinic resources, is painful and is associated with complications: it is important we consider strategies to rationalise presumptive antimicrobial use in MSM and promote antimicrobial stewardship.

We aimed to determine if there are any factors associated with having syphilis among MSM attending as sexual contacts of syphilis. We examined the clinical records of MSM attending as sexual contacts of syphilis from January through December 2019.

Of the 6613 MSM who attended for STI testing, 142/6613 $(2.1 \%)$ presented as sexual contacts of syphilis. The median 